\title{
Retinal dystrophy in long chain 3-hydroxy-acyl-coA dehydrogenase deficiency
}

Iris Schrijver-Wieling, Ger H M B van Rens, Dienke Wittebol-Post, Jan A M Smeitink, Jan P de Jager, Hans B C de Klerk, Godfried H M van Lith

Department of Ophthalmology, University Hospital Utrecht, Utrecht I Schrijver-Wieling D Wittebol-Post

Department of Ophthalmology, Elkerliek Ziekenhuis, Helmond

I Schrijver-Wieling

G H M B van Rens

Department of Metabolic Diseases,

University Children's

Hospital 'Het

Wilhelmina

Kinderziekenhuis',

Utrecht

J A M Smeitink

Department of Pediatrics, Elkerliek Ziekenhuis, Helmond J P de Jager

Department of Pediatrics, Children's Hospital 'Het Sophia Kinderziekenhuis', Rotterdam

H B C de Klerk

Department of Ophthalmology, Oogziekenhuis Rotterdam, Rotterdam, the Netherlands G H M van Lith

Correspondence to: G H M B van Rens, Elkerliek Ziekenhuis, Wesselmanlaan 25, 5707 HA Helmond, the Netherlands.

Accepted for publication 25 November 1996

\begin{abstract}
Background-Long chain 3-hydroxyacyl-CoA dehydrogenase (LCHAD) is one of the enzymes involved in the breakdown of fatty acids. A deficiency of this enzyme is associated with life threatening episodes of hypoketotic hypoglycaemia during prolonged fasting. Neuropathy and retinopigmentary changes were mentioned in only a few cases.
\end{abstract}

Methods-The case histories of two girls, aged 8 and 15 years, with LCHAD deficiency are reported.

Results-Both children with LCHAD deficiency exhibited extensive macular pigmentary depositions and a 'salt and pepper' scattering of pigment in their retinas. The patients have decreasing visual acuity.

Conclusion-The early recognition of LCHAD deficiency can increase the life expectancy in these patients through avoiding catabolism and through appropriate diets. Patients tend to be free of symptoms between attacks, however. Testing for the disorder, therefore, should be included in the diagnostic process for children with retinal dystrophy, in particular when other clinical symptoms are known to have occurred.

(Br F Ophthalmol 1997;81:291-294)

Fatty acids are a major source of metabolic fuel in humans. The oxidation of long chain fatty acids, the most abundant in our daily diets, predominantly occurs in mitochondria. In recent years an increasing number of inherited enzyme defects of fatty acid oxidation such as long chain 3-hydroxy-acyl-CoA dehydrogenase (LCHAD) deficiency have been recognised. ${ }^{1}$ LCHAD is a mitochondrial, membrane bound enzyme that is specific for the long chain substrates. ${ }^{2}$ It dehydrogenates 3-hydroxy-acylCoA compounds of 12 to 18 carbon length, and is multifunctional, also exhibiting 2-enoylCoA hydratase and 3-ketoacyl-CoA thiolase activities. ${ }^{3}$ LCHAD is part of the so called mitochondrial trifunctional protein (MTP), an enzyme complex consisting of four $\alpha$ and four $\beta$ subunits. The $\alpha$ subunit harbours LCHAD activity. ${ }^{5}$ Using fluorescence in situ hybridisation (FISH) the gene for the $\alpha$ subunit was localised to chromosome 2.

Autosomal recessive inheritance is suggested by the intermediate levels of LCHAD activity in the parents of two patients. ${ }^{78}$
Approximately 30 cases of LCHAD deficiency have been reported in the literature. ${ }^{3-17}$ Symptoms are provoked by an insufficient production of ketone bodies during starvation, leading to a severe, life threatening hypoketotic hypoglycaemia. The pattern of organic acids in body fluids at times of stress or during provocative tests, such as prolonged carefully controlled fasting and/or long chain triglyceride loading is indicative of the different enzyme defects involved in fatty acid oxidation. Definitive proof can be obtained by enzyme assays in cultured skin fibroblasts or isolated lymphocytes. ${ }^{12}$ Most patients with LCHAD deficiency have a $\mathrm{G} \rightarrow \mathrm{C}$ mutation at position 1528 of the cDNA encoding the $\alpha$ subunit, resulting in a glutamate to glutamine transition. This base substitution creates a Pst I restriction site making mutation analysis by PCR-RFLP possible. ${ }^{18}$ LCHAD deficiency has been associated with myopathy, cardiomyopathy, intermittent rhabdomyolysis, polyneuropathy, Reye-like syndrome, and sudden unexpected death syndrome. ${ }^{9}{ }^{10} 19$ There can be a sudden onset of symptoms during the first year of life without previous signs of illness. This disease has a high rate of morbidity and mortality.

Very recently retinal dystrophy was shown to be associated with LCHAD deficiency. ${ }^{919}{ }^{20}$ In these reports, the retinal findings were described as retinitis pigmentosa ${ }^{19}$ and pigmentary retinal degeneration. ${ }^{9}$ Until now, detailed ocular findings and the results of electroretinography have not been reported in cases of LCHAD deficiency.

Patients, methods, and results

This paper describes the case histories of two LCHAD deficient girls and focuses on their retinal dystrophy.

The first patient, a girl, was born at term following an uncomplicated pregnancy and delivery in March 1988 as the third child of apparently healthy, non-consanguineous white parents. Her medical history and the biochemical investigations were reported earlier. ${ }^{13}{ }^{14}$ She was examined thoroughly and monitored, because the first child in this family unexpectedly died on the third day after birth. At the age of 5 months she was hospitalised after having suffered from gastrointestinal problems for a few days. Physical examination showed a hypotonic, lethargic, unresponsive, and pale infant with staring eyes. Most probably, these symptoms were caused by the observed hypoketotic hypoglycaemia. During this period of hospitali- 


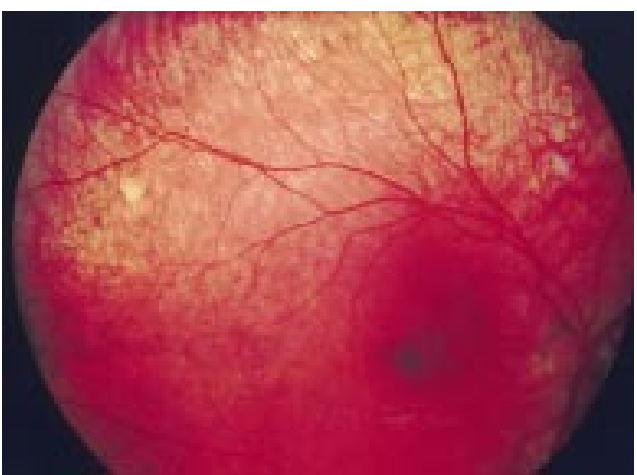

Figure 1 First patient, right eye. Macula with strong hyperpigmentation and some clustering of pigment.

sation, she had two similar episodes and selective screening of body fluids was indicative of a deficiency in fatty acid $\beta$ oxidation, most probably LCHAD. This was confirmed by subsequent enzyme activity measurements. RFLP analysis in this patient revealed the common $\mathrm{G} \rightarrow \mathrm{C} 1528$ mutation in the LCHAD DNA. Treatment was started with a high carbohydrate, medium chain fatty acid enriched diet.

On ophthalmic examination during her first year of life, the girl had a 'salt and pepper' pigmentation in the fundi of both eyes. The electroretinogram was within the normal range, there were no signs of visual impairment, and no strabismus. The next electroretinogram was made in 1993, when she was 5 years old. It shows normal latency time and normal amplitude of the b-wave during both scotopic and photopic conditions.

In October 1994, the child spontaneously complained about decreased vision in the dark. Additional examination of the visual system revealed that visual acuity, with sphere (S) $-0.25=$ cylinder $(\mathrm{C})-1.2590^{\circ}$, was $20 / 25$ in the right eye and, with $\mathrm{S}-0.50=\mathrm{C}-1.2595^{\circ}$, was $20 / 25$ in the left eye. Visual field examination had not yet been performed, in view of the young age of the patient. The diagnosed ocular abnormalities were limited to the fundus. Ophthalmoscopy showed a blond fundus, which is most probably associated with the blond hair and blue eyes of the patient. The papillae were normal and so were the retinal vessels. The appearance of the macula, however, was abnormal in both eyes: it showed strong hyperpigmentation with some clustering of the pigment (Fig 1). In the mid periphery there was an area with atrophy and next to it an arch of salt and pepper pigmentation, changing into a ring of clumped pigment (Fig 2). Salt and pepper pigmentation was visible further into the periphery, while the retina had a virtually normal appearance in the extreme periphery. In addition, there were focal depigmented spots scattered across the entire retina. The left eye showed hyperpigmentation along the edge of the papilla. The vitreous gel was clear.

The second patient, also a girl, was the second child of apparently healthy nonconsanguineous white parents. She was born at term in September 1980 after an uneventful pregnancy and a normal delivery. She devel-

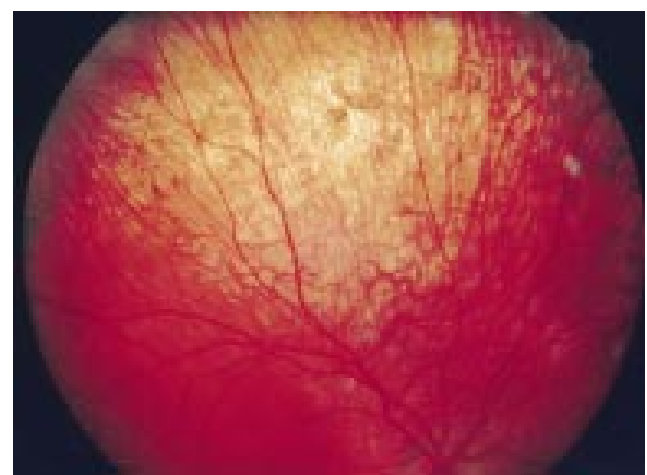

Figure 2 First patient, right eye. Salt and pepper pigmentation, changing into a ring of clumped pigment in the mid periphery.

oped normally until the age of 6 months, when feeding problems and vomiting began. Subsequently, failure to thrive and motor development stagnation caused her to be hospitalised. Physical examination revealed severe muscle weakness and hypotonia. She cried weakly and was pale and lethargic. At that time her weight was $1 \mathrm{~kg}$ below the third percentile expected for her age.

The clinical and chemical findings in connection with the urinary organic acid profile indicated that she was suffering from a deficiency in fatty acid $\beta$ oxidation, and eventually she was diagnosed as having LCHAD deficiency. ${ }^{12}$ RFLP analysis revealed the common $\mathrm{G} \rightarrow \mathrm{C} 1528$ mutation in the LCHAD DNA.

She was examined ophthalmically in 1986. Visual acuity of the right eye was $20 / 20$ with S $-1.0=\mathrm{C}-1.0180^{\circ}$ and that of the left eye $20 / 25$ with $S-1.5=C-1.5180^{\circ}$. Her myopia is most probably because of a familial myopia; her father, for example, is myopic $S-7$. The orthoptic tests and biomicroscopy showed no abnormalities. During ophthalmoscopy, however, hyperpigmentation was found in the periphery as well as in the centre of the retina.

At the age of 10 the patient complained about visual deterioration. She had a reduction in her field of view, decreased vision at dusk and night, as well as photophobia in bright light. With optimal correction, visual acuity of the right eye was still 20/20, but that of the left eye had decreased to 20/40. Ophthalmoscopy revealed pale discs, somewhat narrowed vessels, hyperpigmentation, and pigment clusters in the posterior pole with some sickle-shaped hyperpigmentation in the periphery. Additional examination of the visual system revealed that contrast vision was reduced for the left eye in the middle and high frequencies. Colour vision (Panel tests) was almost normal for the saturated test and highly abnormal in all directions for the unsaturated test version type, especially for the left eye.

The basic value of the electro-oculogram in the dark was normal with a decreased light rise. The EOG Arden ratio was approximately 1.3 and 1.4. In the electroretinogram, amplitudes somewhat less than $50 \%$ of the lower limit of the normal value both for the rod and cone system were found. The pattern evoked occipital potentials were delayed and decreased for 


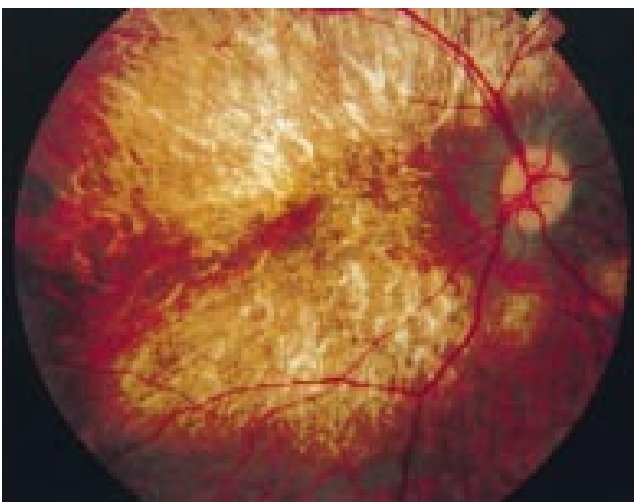

Figure 3 Second patient, right eye. Global view of posterior pole and mid periphery.

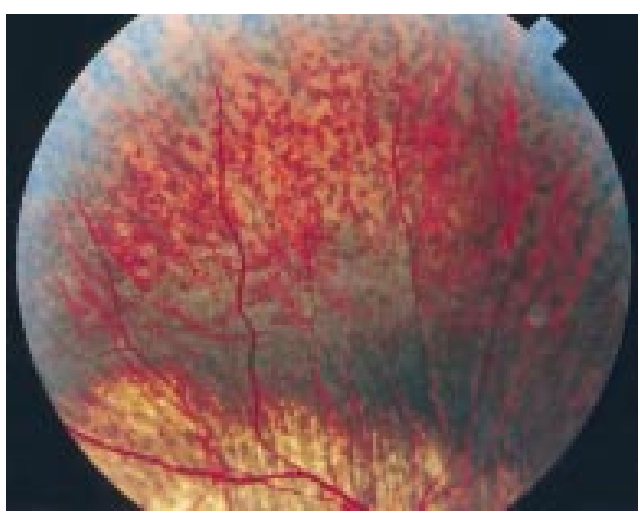

Figure 4 Second patient, right eye. Clumped hyperpigmentation in the periphery.

the left eye, whereas those of the right eye were within the normal range.

At the age of 13 years, visual acuity had further deteriorated-for the right eye it was $20 / 30$ with $\mathrm{S}-7=\mathrm{C}-1.0180^{\circ}$ and for the left eye $3 / 60$ with $S-8.0$. The patient had a central scotoma in the visual field. The ability to see contrast decreased after the test in 1991, while the results of the saturated and unsaturated Panel test of colour vision have not changed significantly. The electroretinograms of the rod and cone system were approximately $75 \%$. The girl was extremely difficult to examine the first time and rather difficult the second time. This probably is the reason for the apparent improvement. A third electrodiagnostic examination was refused by the parents.

At the age of 14, she was seen again. According to the mother, her vision deteriorated and she had more problems at school. Visual acuity for the right and left eye was 20/30 and 2/60 respectively. The vision of $20 / 30$ was achieved with a small central rest in the visual field. The left eye had a deep central scotoma. Ophthalmoscopy showed pale optic discs, hypopigmentation, and hyperpigmentation in the posterior pole (Fig 3) and clumped pigment in the periphery of the retina (Fig 4).

\section{Discussion}

Retinal dystrophy in children may be primary - that is, without other systemic manifestations or secondary and associated either with non-metabolic genetic syndromes or with inherited metabolic disorders. ${ }^{19-21}$ The actual cause and the fundamental pathophysiology of retinal dystrophy are as yet unknown but since they are frequently observed in inherited errors of metabolism it is suspected that they might be induced by toxic effects of certain metabolites, errors of synthetic pathways, or deficient energy metabolism. ${ }^{19}$

One of the inherited disorders of metabolism associated with retinal dystrophy can be caused by deficient LCHAD activity. ${ }^{19}{ }^{20}$ Only recently retinal dystrophy was mentioned in LCHAD deficiency but detailed information about the retinal changes was not given. Electrophysiology was not performed in these studies. ${ }^{9} 1920$

We describe striking retinal dystrophy in two young patients. In our first case, the central part of the retina was affected in the early stages of this metabolic disorder. However, the normal electroretinographic results in both scotopic and photopic conditions did not support a cone-rod degeneration. As yet, the patient has subnormal vision.

If there is destruction of the retinal receptors, the electroretinogram will decrease and eventually become completely extinguished. The implicit time of the b-wave is usually delayed, reflecting dysfunction of the retinal neurons before destruction so that it can be used as an early sign of a progressive disease. A decrease in amplitude reflects the degree of neuronal destruction that may occur somewhat independently of implicit time changes that tend to reflect more diffuse retinal pathology. ${ }^{22}$ In our experience, a rather good electroretinogram compared with fundus changes and visual field abnormalities can sometimes occur in secondary retinal degenerations, as well as in dominantly inherited retinal dystrophies.

A deterioration of the electroretinogram with time appears not to have occurred in the case of the second patient either: her second electroretinogram shows an improvement from $50 \%$ to approximately $75 \%$. We believe, however, that this was not an actual improvement, but rather was caused by the problems experienced while examining the patient during the first test. All other functional tests showed a remarkable deterioration of vision in the left eye and a further deterioration was observed at the age of 14 years. Furthermore, there is a clear increase in retinal dystrophy in the macula and throughout the retina.

The actual cause of the observed retinal dystrophy in LCHAD deficiency is unknown. So far, it is not known if the LCHAD gene is expressed in the pigment epithelium. Whether or not the accumulation of long chain acyl carnitines, observed in LCHAD deficiency, are involved in the pathophysiology of the retinal dystrophy is subject to further investigation.

The treatment of LCHAD deficiency is based on the supplementation of medium chain triglycerides, which can be metabolised normally, as opposed to the long chain triglycerides which cannot. The patients are developing normally on this diet.

Although patients with LCHAD deficiency usually develop symptoms within the first 6 months, these symptoms vary greatly and can be highly non-specific. Early diagnosis, how- 
ever, is very important in view of the life expectancy for these children. In children, LCHAD deficiency and other inherited errors of metabolism should consequently be considered whenever retinal dystrophy is observed.

This article was supported in part by the board of the Elkerliek Ziekenhuis, Helmond, and by the Dr F P Fischer Stichting, Utrecht. We gratefully acknowledge Dr S K Umar-Firting for Utrecht. We gratefully acknowledge Dr S K Umar-Firtina for of the patients.

The data of one patient in this article were, in part, presented

The data of one patient in this article were, in part, presented Toronto, Ontario, Canada, 27 June-2 July 1994

1 Roe CR, Coates PM. Acyl-CoA dehydrogenase deficiencies. In: Scriver CR, Beandit AL, Sly WS, Valle D, eds. The metabolic basis of inherited disease. Vol I. 6th ed. New York: metabolic basis of inherited disease.

2 El-Fakhri M, Middleton B. The existence of an innermembrane-bound, long acyl-chain-specific 3-hydroxyacylCoA dehydrogenase in mammalian mitochondria. Biochim Biophys Acta 1982;713:270-9.

3 Moore R, Glasgow JFT, Bingham MA, Dodge JA, Pollitt RJ, Olpin SE, et al. Long-chain 3-hydroxy-acyl-coenzyme A dehydrogenase deficiency-diagnosis, plasma carnitine fractions and management in a further patient. Eur f Pediatr 1993;152:433-6

4 Carpenter K, Pollitt RJ, Middleton B. Human liver long-chain 3-hydroxyacyl-coenzyme A dehydrogenase is a multifunctional membrane-bound beta-oxidation enzyme of mitochondria. Biochem Biophys Res Commun 1992;183. 443-8.

5 Uchida Y, Izai K, Orii T, Hashimoto T. Novel fatty acid beta-oxidation enzymes in rat liver mitochondria: purification and properties of enoyl-CoA hydratase/3-hydroxyacyltion and properties of enoyl-CoA hydratase/3-hydroxyacylCoA dehydrogenase/3-ketoacyl-CoA thio

6 Ijist L, Ruiter JPN, Jacobs M, Hoovers JMN, Hashimoto T, Wanders RJA. Long-chain 3-hydroxyacyl-CoA dehydrogenase deficiency: mutation analysis, localisation of the gene for the alpha subunit to $2 \mathrm{p} 23.2-2 \mathrm{p} 24.1$ and prenatal diagnosis. Enzyme \& Protein 1995;48:123. (The name of Dr IJlst is misprinted as Ijist.)

7 Jackson S, Bartlett K, Land J, Moxon ER, Pollitt RJ, Leonard JV, Turnbull DM. Long-chain 3- hydroxy-acyl-CoA dehydrogenase deficiency. Pediatr Res 1991;29:406-11.

8 Jackson S, Singh Kler R, Bartlett K, Briggs $\mathrm{H}$, Bindoff LA, Pourfarzam M, et al. Combined enzyme defect of mitochond

9 Bertini E, Dionisi-Vici C, Garavaglia B, Burlina AB, Sabatelli M, Rimoldi M, et al. Peripheral sensory-motor polyneuropathy, pigmentary retinopathy, and fatal cardiomyopathy in long-chain 3-hydroxy-acyl-CoA dehydrogemyopathy in long-chain 3-hydroxy-acyl-CoA
10 Wanders RJA, Duran M, IJlst L, de Jager JP, van Gennip $\mathrm{AH}$, Jakobs C, et al. Sudden infant death and long-chain AH, Jakobs C, et al. Sudden infant death and long-chain

11 Rocchiccioli F, Wanders RJA, Aubourg P, Vianey-Liaud C, IJlst L, Fabre M, et al. Deficiency of long-chain 3-hydroxyacyl-CoA dehydrogenase: a cause of lethal myopathy and cardiomyopathy in early childhood. Pediatr Res 1990;28: 657-62.

12 Przyrembel H, Jakobs C, IJlst L, de Klerk JBC, Wanders RJA. Long-chain 3-hydroxy-acyl-CoA dehydrogenase deficiency. F Inherited Metab Dis 1991;14:674-80.

13 Wanders RJA, IJlst L, van Gennip AH, Jakobs C, de Jager JP, Dorland L, et al. Long-chain 3-hydroxy-acyl-CoA dehydrogenase deficiency: identification of a new inborn error of genase deficiency: identification of a new inborn error of
mitochondrial fatty acid beta-oxidation. F Inherited Metab Dis 1990;13:311-4

14 Duran M, Wanders RJA, de Jager JP, Dorland L, Bruinvis L, Ketting D, et al.3-Hydroxydicarboxylic aciduria due to ong-chain 3-hydroxy-acyl-coenzyme A dehydrogenase deficiency associated with sudden neonatal death: protective effect of medium-chain triglyceride treatment. Eur $\mathcal{F}$ Pediatr 1991;150:190-5.

15 Dionisi-Vici C, Burlina AB, Bertini E, Bachmann C, Mazziotta MRM, Zacchello F, et al. Progressive neuropathy and recurrent myoglobinuria in a child with long-chain 3-hydroxy-acyl-coenzyme A dehydrogenase deficiency. $f$ Pediatr 1991;118:744-6.

16 Dionisi-Vici C, Bertini E, Burlina A, Garavaglia A, Hale $\mathrm{DE}$, Bartuli A, et al. Neuromuscular involvement in two unrelated children with long-chain 3-hydroxy-acyl-CoA dehydrogenase (LCHAD) deficiency. Pediatr Res 1990;28: dehy 305 .

17 Hale DE, Thorphe C, Braat K, Wright JH, Roe CR, Coates $\mathrm{PM}$, et al. The L-3-hydroxyacyl-CoA dehydrogenase deficiency. In: Tanaka K, Coates PM, eds. Fatty acid oxidation, clinical, biochemical and molecular aspects. New York: Alan R Liss, 1990:503-10.

18 Ylst L, Wanders RJA, Ushikubo S, Kamijo T, Hashimoto T. Molecular basis of long-chain 3-hydroxyacyl-CoA dehycrousing mutation in the alpha-subunit of the major diseasetrifunctional protein. Biochim Biophys Acta 1994;1215:34750.

19 Poll-The BT, Billette de Villemeur T, Abitbol M, Dufier JL, Saudubray JM. Metabolic pigmentary retinopathies: diagnosis and therapeutic attempts. Eur F Pediatr 1992;151:211

20 von Döbeln U, Nyberg G, Hagenfeldt L. Clinical symptoms and dietary treatment of 3-hydroxyacyl-CoAdehydrogenase deficiency. Proceedings of the VI th International congress Inborn Errors of Metabolism, Milan, Italy, 27-31 May 1994:239.

21 Moore A. Inherited retinal dystrophies. In: Taylor D, ed. Pediatric ophthalmology. Cambridge: Blackwell Scientific, 1990:376-403.

22 Stanescu B, Michiels J, Lyon G. Electroretinography (ERG) and hereditary transmission in neurologic heredodegenerative diseases. Birth Defects Original Article Series 1982;18:255-62. 